Then the energy factors in Eq. (11) can be written as

$$
\frac{\left(E_{2 s}-E_{n}\right)\left(E_{n}-E_{1 s}\right)}{\omega_{1} \omega_{2}\left(E_{n}-E_{1 s}-\omega_{1}\right)}=\frac{E_{2 s}-E_{n}}{\omega_{1} \omega_{2}}-\frac{1}{\omega_{2}}+\frac{1+\Delta / \omega_{2}}{E_{n}-E_{1 s}-\omega_{1}},
$$

and the exchange term can be written as

$$
\frac{\left(E_{2 s}-E_{n}\right)\left(E_{n}-E_{1 s}\right)}{\omega_{1} \omega_{2}\left(E_{n}-E_{1 s}-\omega_{2}\right)}=\frac{E_{1 s}-E_{n}}{\omega_{1} \omega_{2}}+\frac{1}{\omega_{2}}+\frac{\Delta}{\omega_{1} \omega_{2}}+\frac{1+\Delta / \omega_{1}}{E_{n}-E_{1 s}-\omega_{2}} .
$$

When these expressions are substituted into Eq. (11) the result is

$$
M_{p}=M_{r}+\left(1+P_{12}\right) \frac{\Delta}{\omega_{2}} \sum_{n} \frac{\left\langle 2 s\left|e \overrightarrow{\mathrm{E}}_{2} \cdot \overrightarrow{\mathrm{r}}\right| n\right\rangle\left\langle n\left|e \overrightarrow{\mathrm{E}}_{1} \cdot \overrightarrow{\mathrm{r}}\right| 1 s\right\rangle}{E_{n}-E_{1 s}-\omega_{1}}+\frac{\Delta}{\omega_{1} \omega_{2}}\left\langle 2 s\left|\left(e \overrightarrow{\mathrm{E}}_{1} \cdot \overrightarrow{\mathrm{r}}\right)\left(e \overrightarrow{\mathrm{E}_{2}} \cdot \overrightarrow{\mathrm{r}}\right)\right| 1 s\right\rangle .
$$

The first term on the right-hand side of Eqs. (13) and (14) combine in Eq. (11) to give zero, when the completeness and orthogonality of the eigenstates of $H_{0}$ are used, along with the commutation relations. Equation (15) shows that for nonresonance, $\Delta \neq 0$, the matrix element $M_{p}$ is not equal to $M_{r}$. For resonance,

$$
M_{p}=M_{r} \text { for } \Delta=0 \text {. }
$$

Thus, in the case of resonant two-photon absorption, either interaction may be used, ${ }^{8}$ but not in the case of nonresonance. The same conclusions hold for the absorption of radiation by a two-level atom. ${ }^{2}$

The example given here shows that, contrary to a widespread opinion, ${ }^{9}$ it does make a difference in some problems whether the $\overrightarrow{\mathrm{E}} \cdot \overrightarrow{\mathrm{r}}$ interaction or the $\vec{A} \cdot \vec{p}$ interaction is used. ${ }^{10}$ Correct results will always be obtained in problems in which the electric-dipole approximation can be made if the $\overrightarrow{\mathrm{E}} \cdot \overrightarrow{\mathrm{r}}$ interaction is used.

I would like to thank Dr. Arthur L. Smirl and Dr. Ahmet Elci for discussions and for reading the manuscript. This work was supported in part by a grant from the North Texas State Uni-

\section{versity Faculty Research Fund.}

${ }^{1}$ K.-H. Yang, Ann. Phys. (N.Y.) 101, 62 (1976).

${ }^{2} \mathrm{D} . \mathrm{H}$. Kobe and A。 L。 Smirl, to be published。

${ }^{3}$ J. J. Forney, A. Quattropani, and F. Bassani, Nuovo Cimento $37 \mathrm{~B}, 78$ (1977)。

${ }^{4}$ W. E. Lamb, Jr., Phys. Rev。 85, 259 (1952).

${ }^{5}$ F. Bassani, J. J. Forney, and A. Quattropani, Phys. Rev. Lett。39, 1070 (1977).

${ }^{6}$ The $A^{2}$ term contributes only to the higher multipole interactions. See, e.g., R。 Guccione and J。Van Kranendonk, Phys. Rev. Lett. 14, 583 (1965)。

${ }^{7}$ B. Cagnac, G. Grynberg, and F. Biraben, J. Phys。 (Paris) 34, 845 (1973).

${ }^{8}$ Similar conclusions for the resonant case have been obtained for the Kramers-Heisenberg dispersion formula. Cf. W. P. Healy, Phys. Rev. A 16, 1568 (1977).

${ }^{9}$ See, e.g., A. Gold, in Quantum Optics, edited by R. J. Glauber (Academic, New York, 1969), p. 397 (cf.p。 402 in particular).

${ }^{10} \mathrm{This}$ difference is not only the practical difference of rate of convergence suggested in Ref. 5. See also J. M. Worlock, in Laser Handbook, edited by F. T. Arecchi and E. O. Schulz-Dubois (North-Holland, Amsterdam, 1972), Vol. 2, p. 1323 (cf。pp.13271328).

\title{
Spectroscopy of Electronic States in Superheavy Quasimolecules
}

\author{
Gerhard Soff, Berndt Müller, and Walter Greiner \\ Institut für Theoretische Physik der Johann Wolfgang Goethe-Universität, Frankfurt am Main, Germany
} (Received 26 August 1977)

\begin{abstract}
We show that information about quasimolecular electronic binding energies in transient atomic systems of $Z=Z_{1}+Z_{2}$ up to 184 can be obtained from three sources: (1) the impact-parameter dependence of the ionization probability; (2) the ionization probability in head-on collisions as a function of total nuclear charge $Z$; (3) the delta-electron spectrum in coincidence with $K$-vacancy formation in asymmetric collisions. Experiments are proposed and discussed.
\end{abstract}

In collisions of very heavy ions $Z_{1}$ and $Z_{2}$ it is possible to form quasimolecular systems where the binding energy of the inner-shell electrons is of the order of the electron mass. These systems represent a prototype of highly relativistic, strongly bound quantum systems and have there- 
fore attracted considerable theoretical interest over recent years., ${ }^{1,2}$ A determination of the binding energies in such quasimolecular systems would fill in the gap between the normal atoms and the still poorly understood nuclear and subnuclear systems in which binding is mediated by the strong interaction.

The experimental investigation meets with two difficulties: (1) The short lifetime of a quasimolecule is related to a large uncertainty in the energy of the electronic states; (2) the direct measurement of transition energies by $\mathrm{x}$-ray spectroscopy is obstructed by the high nuclear $\gamma$-ray background following nuclear Coulomb excitation except for $\mathrm{Pb}$ or $\mathrm{Bi}$ ions. The nuclear background will also make measurements of the $\mathrm{x}$-ray anisotropy ${ }^{3}$ for transitions to the $1 s \sigma$ state impossible. In this Letter we show how to use the time dependence of the quasimolecular states for the measurement of binding energies, without looking at the $x$-ray channel.

Before we enter the quantitative discussion, let us state something about the possible accuracy of the measurements. The energy uncertainty causes the electrons to be distributed over a broad spectrum of states leading to ionization of innershell electrons. Since the time variation of the molecular states is known precisely, the energy spectrum of ejected electrons can be calculated and the binding energy of the state from which the electrons originate can be inferred. In principle, a precision of the order of $10 \%$ is attainable in coincidence measurements of ionization probabilities. ${ }^{4}$ This would allow for a check of the predicted inner-shell binding energies within an accuracy of $20 \mathrm{keV}$. This is just on the verge of ordinary quantum-electrodynamic effects such as vacuum polarization. It would certainly suffice to detect any serious deviation from the predicted values of binding energy.

We calculate the direct ionization into the electron continuum $\left(E>m c^{2}\right)$ in first-order time-dependent perturbation theory, restricting ourselves to the most important radial coupling. ${ }^{5}$ The transition amplitude is expressed as

$$
\begin{aligned}
a(E)=- & \int_{-\infty}^{\infty} d t \dot{R}(t)\left\langle\psi_{c s}(E)|(\partial / \partial R)| \psi_{b s}\right\rangle \\
& \times \exp \left\{i \int_{-\infty}^{t} d t^{\prime}\left[E_{c s}-E_{b s}\left(t^{\prime}\right)\right]\right\} .
\end{aligned}
$$

$\dot{R}$ denotes the ion velocity given by the Rutherford trajectory. In the wave functions $\psi_{c s}(E)$ and $\psi_{b s}$ we take into account only the monopole term $V_{\mathrm{MP}}(r, R)=(4 \pi)^{-1} \int d \Omega V_{\mathrm{TC}}(\overrightarrow{\mathrm{r}}, R)$ of the multipole expansion of the exact two-center potential
$V_{\mathrm{TC}}(\overrightarrow{\mathrm{r}}, R)$. Binding energies of the $1 s \sigma, 2 s \sigma, 3 s \sigma$, and $2 p_{1 / 2} \sigma$ states and radial matrix elements calculated in this monopole approximation are in agreement to better than $5 \%$ when compared with exact two-center calculations up to distances $R$ $\sim 500 \mathrm{fm}$ which are most important for direct ionization. ${ }^{5}$

The total ionization cross section is given by

$$
\begin{aligned}
\sigma & =2 \pi \int_{0}^{\infty} b d b P(b) \\
& \equiv 2 \pi \int_{0}^{\infty} b d b\left(2 j_{i}+1\right) \int_{m_{e^{c}}{ }^{2}}^{\infty} d E|a(E)|^{2},
\end{aligned}
$$

where the statistical factor $\left(2 j_{i}+1\right)$ accounts for the occupation number of the initial state.

Figure 1 shows the number of $1 s \sigma$ vacancies per collision, $P(b)$, as a function of the impact parameter $b$ for the superheavy quasimolecules $Z=140,164$, and 184, calculated from Eqs. (1) and (2). The projectile energy was chosen such that the distance of closest approach is $R_{\text {min }}=20$ $\mathrm{fm}$ in each case. For $b \gtrsim 20 \mathrm{fm}, P(b)$ falls off exponentially ${ }^{5-7}$ :

$$
P(b) \sim \exp (-b / a),
$$

the fall-off constant $a(Z)$ varying from $a(140)=27$ $\mathrm{fm}$ to $a(164)=18 \mathrm{fm}$ and $a(184)=17 \mathrm{fm}$. It is important to note that the ionization probability deviates from an exponential behavior for $b<20$ $\mathrm{fm}$ where it becomes nearly a constant. Analytical considerations have led to the conclusion

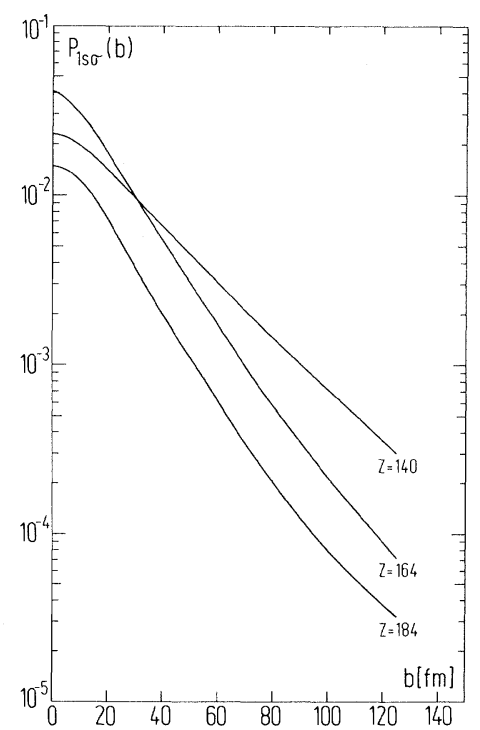

FIG. 1. Impact-parameter dependence of the number $\boldsymbol{P}(b)$ of $1 s \sigma$ vacancies per collision produced via radial coupling for $Z=140,164$, and 184. $R_{\min }=20 \mathrm{fm}$ determines the projectile energy. $P(b)$ is normalized to 2 . 
that $P(b)$ can be represented with good accuracy by the following expression ${ }^{8}$ :

$$
P(b)=D(Z) \exp \left[-E_{b}\left(R_{0}\right) / \Gamma\right] .
$$

Here $R_{0}(b)$ is the distance of closest approach on a given Coulomb trajectory with center-of-mass energy $E_{\text {c.m. }}, E_{b}(R)>0$ is the $1 s \sigma$ binding energy, $v$ is the ion velocity at infinity, and

$$
\Gamma(b)=\frac{\hbar v}{4 R_{0}(b)}\left[2-\frac{Z_{1} Z_{2} e^{2}}{R_{0} E_{\mathrm{c} . \mathrm{m} .}}\right]^{1 / 2} .
$$

The function $D(Z)$ is given by

$$
D=4.30 \exp \left(-Z^{\prime} / 12.9\right)\left[1+\exp \left(-Z^{\prime} / 7.7\right)\right]^{-1}
$$

$\left(Z^{\prime}=184-Z\right)$. The main feature of formula (4) is the one-to-one correspondence between the binding energy $E_{b}\left(R_{0}\right)$ of the $1 s \sigma$ level at the distance of closest approach and the impact-parameter dependence of the ionization probability $P(b)$ which can be measured experimentally. The falloff constant $\Gamma$ has a typical value of $200 \mathrm{keV}$ for $b=0$. An uncertainty $\Delta P$ in the measurement of $P(b)$ can be directly related to the uncertainty $\Delta E$ of the $E_{b}\left(R_{0}\right)$ thus deduced, giving the numbers quoted above. It should be noted that an error in $D(Z)$, which could result from the rather complicated numerical computation, only leads to a uni form shift of the energy curve $E_{b}\left(R_{0}\right)$, but leaves the shape unaltered. Also, since $R_{0}(b) \sim b$ for large $b, \Gamma \sim 1 / b$ and $P(b)$ in Eq. (4) will take the exponential form (3) for large impact parameters. We see from Eqs. (3)-(5) that the value of the constant $a$ is related to the binding energy of the state to be ionized.

For the direct $2 p_{\sqrt{ } / 2} \sigma$ ionization via radial coupling, qualitatively similar results hold. The impact-parameter dependence is only half as steep as that for the $1 s \sigma$ state. As we have seen, this fact reflects the smaller binding energy of the $2 p_{1 / 2} \sigma$ state. However, one has to realize that rotational coupling between the $2 p_{1 / 2} \sigma$ state and higher bound states may modify the calculated impact-parameter dependence and also may lead to additional contributions for the total cross section.

In order to support the strong relation between the impact-parameter dependence of ionization and the binding energy of the initial state, we have artifically lowered the binding energy $E_{b}(R)$ in our numerical code to $E_{b}(R)-\Delta E(R)$. In $\mathrm{Pb}$ $+\mathrm{Pb}$ we have chosen $\Delta E(R)=(200 \mathrm{keV})[1-R /$ $(1000 \mathrm{fm})]$ and $\Delta E=0$ for $R>1000 \mathrm{fm}$. In a collision with $R_{\min }=16 \mathrm{fm}, P(b=0)$ increases from
0.085 to 0.15 and the falloff constant $a$ from 19 to $31 \mathrm{fm}$. Such changes are far outside of even the present experimental uncertainty.

For symmetric collisions with $136 \leqslant Z_{1}+Z_{2} \leqslant 184$ the number of vacancies produced per collision in the $1 s \sigma$ shell at $b=0$ is shown in Fig. 2(a) for different ion energies characterized by the distance of closest approach. The increase for $Z$ $<160$ is due to the rapidly growing localization of the $1 s \sigma$ wave function in the range $130<Z<160$ (the so-called collapse to the center). For higher total charges the binding energy of the $K$ electrons increases strongly (see, e.g., Greenberg et $\left.a l .{ }^{9}\right)$. Then larger Fourier frequencies are needed for ionization which are not available in the collision. Hence the ionization probability is reduced again. The measurement of $P(b=0)$, which is experimentally possible, ${ }^{10,11}$ would prove for the first time the strong electronic binding for $Z>160$ as theoretically predicted. ${ }^{9}$ The maximum of $P(b=0)$ shifts from $Z=168$ to $Z=154$ if one changes the distance of closest approach
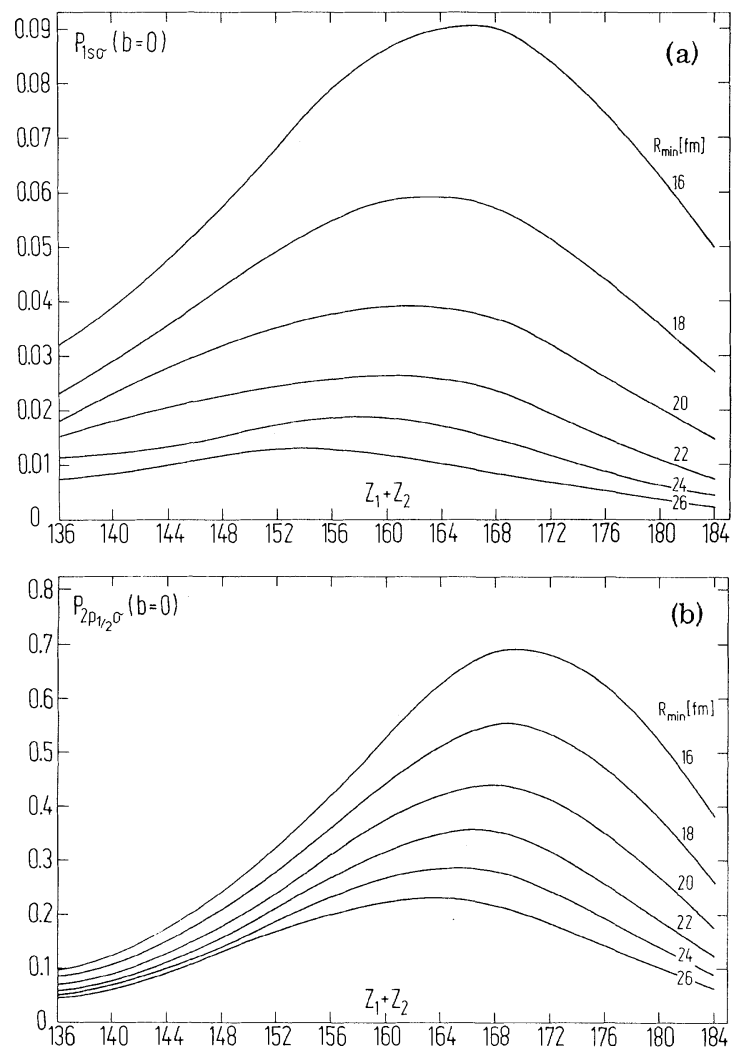

FIG. 2. (a) The number of $1 s \sigma$ vacancies per collision for head-on collisions as function or the total charge $Z_{1}+Z_{2}$ for different ion energies. (b) The same for $2 p_{1 / 2} \sigma$ ionization. 
from $R_{\text {min }}=16$ to $26 \mathrm{fm}$. No maximum is found for low ion energies $\left(R_{\text {min }}>35 \mathrm{fm}\right)$. This is understandable because in such distant collisions the united-atom limit cannot be reached.

Similar effects are found for the $2 p_{1 / 2} \sigma$ level. $P(b=0)$ for $2 p_{1 / 2} \sigma$ ionization via radial coupling [Fig. 2(b)] increases roughly by one order of magnitude from $Z=136$ to 168 and decreases again by a factor of 2 at $Z=184$ as a result of the strong increase in binding energy, which exceeds even that of the $1 s \sigma$ level. $2 p_{1 / 2} \sigma$ ionization for headon collisions can be measured using $U$ as the projectile and heavy targets like $\mathrm{Au}, \mathrm{Bi}$, or $\mathrm{Pb}$, whereas more asymmetric systems are preferable for $1 s \sigma$ experiments in order to evade the $1 s \sigma-2 p \sigma$ vacancy-sharing process. Such measurements would allow us to investigate the $Z$ dependence of binding energies in the region of superheavy elements.

For the sake of completeness we show in Fig. 3 the number of vacancies produced per collision at $b=0$ for the $\mathrm{Pb}-\mathrm{Pb}$ system as a function of the ion energy $E_{1 \mathrm{ab}}$ for both $1 s \sigma$ and $2 p_{1 / 2} \sigma$ ionization. For low ion energies an exponential increase is found. A comparison with Eq. (4) may be used to obtain information on the binding energy at the distance of closest approach, $E_{b}\left(R_{0}\right)$. This would be equivalent to a $P(b)$ measurement at fixed scattering energy.

Finally, we wish to discuss the spectrum of the ionized electrons. Details calculations for $\mathrm{Pb}$

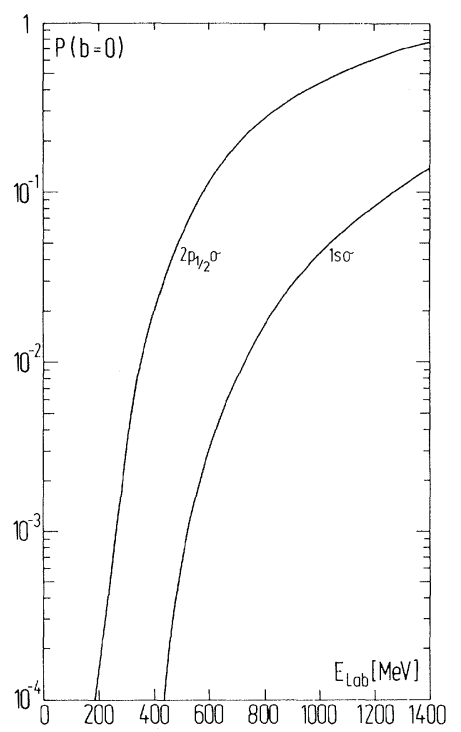

FIG. 3. The number of vacancies in the $1 s \sigma$ and $2 p_{1 / 2} \sigma$ states for head-on collisions in the $\mathrm{Pb}-\mathrm{Pb}$ quasimolecule as a function of projectile energy.
$+\mathrm{Pb}$ collisions are being published elsewhere. ${ }^{6}$ The radial density distribution of the ionized state is reflected in the high-energy part ( $E$ $z 2000 \mathrm{keV}$ ) of the electron spectrum, which follows from very central collisions ( $b \lesssim 10 \mathrm{fm}$ ) only. Therefore, the spectrum could be thought to be a good source for information about the short-distance behavior of the $1 s \sigma$ wave function. However, because of similar form factor and a smaller energy gap, the high-energy electron spectrum resulting from $2 p_{1 / 2} \sigma$ ionization is larger than that from $1 s \sigma$ ionization (for $Z>140$ ). Therefore a spectroscopy of the $1 s \sigma$ shell for these systems by investigating the distribution of the continuum electrons must be performed on asymmetric systems, where the high-energy electron spectrum and the $K$ x rays of the heavier collision partner (containing no vacancy-sharing contribution) can be measured in coincidence.

Because of the excellent agreement between our calculation and first experimental results r $^{40,11}$ on the impact-parameter dependence of innershell ionization in superheavy quasimolecules we are finally led to the conclusion that it should be possible to deduce binding energies with an accuracy of less than $20 \mathrm{keV}$. This number follows from $\Delta E=\Gamma(b) \Delta P / P(b)$ with $\Gamma(b) \sim 200 \mathrm{keV}$ and $\Delta P / P(b) \sim 0.1$ (Ref. 4 ), which is the uncertainty in the measurement of the ionization probability. Although in a somewhat indirect way, a full-scale spectroscopy of superheavy atomic states will result, giving us valuable insight into the physics of very tightly bound, highly relativistic systems.

We acknowledge fruitful discussions with Professor J. S. Greenberg, Professor E. Merzbacher, and Professor W. Meyerhof. This work was supported by the Bundesministerium für Forschung und Technologie (BMFT) and by the Gesellschaft für Schwerionenforschung (GSI).

\footnotetext{
${ }^{1}$ See, e.g., J。 Reinhardt and W. Greiner, Rep. Prog. Phys. 40, 219 (1977).

${ }^{2}$ W. Meyerhof, Science 193, 839 (1976).

${ }^{3}$ C. Stoller, W. Wölfli, G. Bonani, M. Stöckli, and M. Suter, J. Phys. B 10, L347 (1977).

${ }^{4}$ J. R. Macdonald, P. Armbruster, H. H. Behncke, F. Folkmann, S. Hagmann, D. Liesen, P. H. Mokler, and A. Warczak, Z. Phys. A 284, 57 (1978).

${ }^{5}$ W. Betz, G. Soff, B. Müller, and W. Greiner, Phys. Rev. Lett. 37, 1046 (1976).

${ }^{6}$ D. H. Jakubassa, Phys. Lett. $\underline{58 \text { A, }} 163$ (1976).
} 
${ }^{7}$ G. Soff, W. Betz, B. Müller, W. Greiner, and $\mathrm{E}$. Merzbacher, "Inner-Shell Ionizationin the $\mathrm{Pb}-\mathrm{Pb}$ Quasimolecule" (to be published).

${ }^{8}$ B. Muller, G. Soff, W. Greiner, and V. Ceausescu, "Scaling Behavior of Inner-Shell Ionization in Superheavy Quasimolecules" (to be published).

${ }^{9}$ W. Pieper and W. Greiner, Z. Phys. 218, 327 (1969).
${ }^{10}$ J. S. Greenberg, H. Bokemeyer, H. Emling, E. Grosse, D. Schwalm, and F. Bosch, Phys. Rev. Lett. 39, 1404 (1977).

${ }^{11} \mathrm{R}$. Anholt and W. Meyerhof, "Probability of Forming $1 s \sigma$ Vacancies in $625-\mathrm{MeV} \mathrm{Xe}+\mathrm{Au}, \mathrm{Pb}, \mathrm{Bi}$, and $\mathrm{Th}$ Collisions at Small Impact Parameters" (to be published).

\title{
Interaction between a Spatially Extended Collection of Two-Level Systems and a Resonant Radiation Mode
}

\author{
Abraham Shalom ${ }^{(a)}$ \\ Department of Physics, Technion-Israel Institute of Technology, Haifa, Israel \\ (Received 3 October 1977)
}

\begin{abstract}
This paper reports on the analysis of a spatially extended collection of two-level systems inside a cavity filled with electromagnetic radiation. A new feature of the analysis is the consideration of different strengths of coupling between different atoms and the field (due to the variation of the field intensity inside the cavity). The results are qualitatively different from those obtained under the assumption of equal coupling for all atoms.
\end{abstract}

The interaction of a number of two-energy-level systems (TLS's) with the electromagnetic field has received much attention in the literature. ${ }^{1}$ Emission of radiation into free space by a collection of TLS's has been investigated by numerous authors both in the case where the TLS's are confined $^{1,2}$ to a small spatial region (in comparison with the wavelength of the emitted radiation), and in the case $\mathrm{c}^{3,4}$ of larger spatial regions neglecting the reaction of the field on the TLS's. For the problem of emission inside a cavity, as encountered in theoretical $1^{5}$ laser models, the field reaction is important and cannot be neglected. Previous investigations ${ }^{1,6}$ have concentrated on the case where the TLS's are confined to a small region and couple equally with the field. It is the purpose of the present paper to analyze the behavior of a spatially extended collection of TLS's inside the cavity. It is shown that the totalsystem behavior is qualitatively different from that of a localized collection and also different from that predicted by a previous approach ${ }^{7}$ to the same problem.

My system consists of a number of TLS's and a resonant radiation mode of angular frequency $\omega$. The $j$ th TLS is described by three angularmomentum operators $l_{1}(j), l_{2}(j)$, and $l_{3}(j)$, satisfying $\left[l_{1},(j), l_{2}(j)\right]=i l_{3}(j)$. The electric and magnetic fields are given respectively by $\overrightarrow{\mathrm{E}}(\overrightarrow{\mathrm{r}}, t)$ $=-(4 \pi \hbar \omega)^{1 / 2} \overrightarrow{\mathrm{u}}(\overrightarrow{\mathrm{r}}) p(t)$ and $\overrightarrow{\mathrm{B}}(\overrightarrow{\mathrm{r}}, t)=\left(4 \pi c^{2} \hbar / \omega\right)^{1 / 2} \nabla$ $\times \overrightarrow{\mathrm{u}}(\overrightarrow{\mathrm{r}}) q(t), \quad \overrightarrow{\mathrm{u}}(\overrightarrow{\mathrm{r}})$ satisfies $\nabla^{2} \overrightarrow{\mathrm{u}}(\overrightarrow{\mathrm{r}})+\left(\omega^{2} / c^{2}\right) \overrightarrow{\mathrm{u}}(\overrightarrow{\mathrm{r}})=0$ and is normalized over the cavity volume; $q$ and $p$ are dimensionless variables satisfying $[q, p]=i$. The Hamiltonian is given (using the rotating-wave and electric-dipole approximations) by

$$
\begin{aligned}
H=\hbar \omega\left(a^{\dagger} a+\frac{1}{2}\right) & +\hbar \omega \sum_{j} l_{3}(j) \\
& +\frac{1}{2} \hbar \sum_{j} g(j)\left[a^{\dagger} l_{-}(j)+a l_{+}(j)\right],
\end{aligned}
$$

where $a=2^{-1 / 2}(q+i p), a^{\dagger}=2^{-1 / 2}(q-i p),\left[a^{\dagger}, a\right]=1$, and $l_{ \pm}(j)=2^{-1 / 2}\left[l_{1}(j) \pm l_{2}(j)\right]$. The coupling constant is given by $g(j)=(4 \pi \omega / \hbar)^{1 / 2} \vec{\mu} \cdot \overrightarrow{\mathrm{u}}\left(\overrightarrow{\mathrm{r}}_{j}\right) ; \vec{\mu}$ is the transition dipole moment taken to be real; and $\vec{r}_{j}$ is the position of the $j$ th TLS in the cavity. Take $\overrightarrow{\mathrm{u}}(\overrightarrow{\mathrm{r}})$ to be a standing wave depending on one spatial coordinate, ${ }^{5} \overrightarrow{\mathrm{u}}(\overrightarrow{\mathrm{r}})=\overrightarrow{\mathrm{u}}(0) \sin (\omega x / c)$. Thus

$$
g(j)=g \sin \left(\omega x_{j} / c\right) ; \quad g=g\left(x_{j}=c \pi / 2 \omega\right) .
$$

All TLS's having a certain $x$ coordinate or an $x$ coordinate differing from it by a multiple of one quarter-wavelength, $\frac{1}{4} \lambda=\pi c / 2 \omega$, have equal coupling constants. All such TLS's may be combined into a single quantum mechanical system, which has been ${ }^{2,6}$ referred to as angular momentum oscillator (AMO). Each AMO is described by angular-momentum components (equal to the algebraic sums of those of its constituent TLS's), and a total angular-momentum quantum number $L_{0^{*}}$ Let us assume that all TLS's are excited initially so that $L_{0}$ has its maximal value, i.e., one half times the number of TLS's combined into the AMO; thus, $L_{0}$ is a macroscopic number. The summation in Eq. (1) is now to be understood as summation over AMO's. 